\title{
Caracterização Morfológica e Hidrodinâmica de Praias do Estado de Santa Catarina com Vistas à Avaliação de Perigo ao Banhista
}

\author{
Raphael Souza Ribeiro* \\ Jarbas Bonetti** \\ Antonio Henrique da Fontoura Klein ${ }^{* * *}$
}

\section{Resumo}

O objetivo deste trabalho foi avaliar o grau de perigo aos banhistas em 14 praias do litoral do Estado de Santa Catarina. Para isso foi utilizada a metodologia aplicada aos planos de segurança das praias australianas, que se baseia no cálculo o grau de perigo com base no estágio morfodinâmico e na altura de quebra de ondas da praia. As praias avaliadas foram escolhidas com base no número de ocorrências registradas pelo corpo de bombeiros do estado. A classificação do estágio morfodinâmico das praias foi realizada a partir da análise de fotografias aéreas e imagens de satélite e os dados de altura de onda foram obtidos da base de dados do projeto SMC Brasil. Dentre as praias analisadas houve predomínio do estágio intermediário, com apenas uma praia refletiva e nenhuma praia dissipativa. Os graus de risco variaram de baixo a moderado nestas praias.

Palavras-chave: Segurança de praias; Estágio morfodinâmico; Altura de quebra de onda.

** Mestrando do PPGG - CFH/UFSC (rapaheoceano@gmail.com).

***** Professor do Departamento de Geociências e do Programa de PósGraduação e Oceanografia - CFH/UFSC (jarbas.bonetti@ufsc.br).

****** Professor do Departamento de Geociências e do Programa de PósGraduação e Oceanografia - CFH/UFSC (ahfklein@gmail.com).

Geosul, Florianópolis, v. 30, n. 60, p 49-68, jul./dez. 2015 
RIBEIRO, R.S et al. Caracterização morfológica e hidrodinâmica de praias...

Morphological and Hydrodynamic Characterization of Santa Catarina State Beaches for the Assessment of Risk Level to the Bather

\begin{abstract}
The main goal of this study was to evaluate the risk level to bathers on 14 beaches of Santa Catarina State coast. For this, we used the methodology applied on Australian beach safety plans, which consists on calculating the hazard rate based on the morphodynamic beach type and wave breaking height. The evaluated beaches were chosen based on the number of incidents with bathers recorded by the state's fire department (responsible for beach safety). The morphodynamic stage classification of beaches was undertook from the analysis of aerial photographs and satellite imagery and wave height data were obtained from the database of SMC Brazil project. Among the beaches studied in this research, the intermediate state was dominant with only one reflective and no dissipative beach. The risk levels ranged from low to moderate.
\end{abstract}

Key words: Peach safety; Morphodynamic beach state; Wavebreaking height.

\title{
Introdução
}

Nas últimas décadas o ser-humano vem ocupando cada vez mais intensamente as áreas costeiras e, consequentemente, utilizando as praias arenosas oceânicas como áreas de recreação e turismo. A crescente demanda por tais usos nos municípios litorâneos induziu, muitas vezes, um desenvolvimento com baixo grau de planejamento, desconsiderando a natureza móvel e dinâmica da linha de costa e gerando efeitos adversos.

Segundo Spilman (2000), a cada ano morrem no mundo cerca de 490 mil pessoas em decorrência de afogamentos. No Brasil, o afogamento está em primeiro lugar como causa de morte 
RIBEIRO, R.S et al. Caracterização morfológica e hidrodinâmica de praias...

entre a faixa etária de 5 a 39 anos. Os números crescem no verão, estação em que há maior quantidade de banhistas nas praias.

Hoefel \& Klein (1998) ressaltaram a importância da relação entre os determinantes sociais e ambientais para a análise da segurança em relação ao banhista. Mocellin (2006), por seu turno, observou que variantes ambientais como tipo de ondulação, altura de onda e posição das correntes de retorno, em associação ao número de frequentadores da praia, estão diretamente relacionadas ao volume de acidentes ocorridos com banhistas.

Atento a este problema, desde 1990 o governo australiano, através do programa The Australian Beach Safety and Management Program (ABSAMP), avalia as características, perigos e níveis de risco das praias do país utilizando a metodologia proposta por Short (2002). No Brasil, dentre as iniciativas para minimizar o número de fatalidades por afogamento o Projeto "Gerenciamento e Segurança nas Praias Catarinenses" desenvolvido pelo Corpo de Bombeiros de Santa Catarina em conjunto com a Universidade do Vale do Itajaí (UNIVALI) tornou-se umas das principais referências sobre o assunto. Teve início em 1995 com o objetivo de desenvolver uma base de dados sobre as características físicas e socioeconômicas das praias catarinenses, definir o grau de periculosidade das praias do estado e formular um programa de orientação ao seu público usuário (KLEIN \& MEDEIROS, 1998).

O presente trabalho visa dar continuidade a esse tipo de estudo. Nele, a classificação morfodinâmica e a determinação da altura de onda incidente, definidos para praias que apresentam elevados registros de incidentes com banhistas, permitiram que se avaliasse de forma quantitativa o risco em diferentes praias do Estado de Santa Catarina.

\section{Elementos dinâmicos área de estudo}

O Estado de Santa Catarina apresenta regime de micromarés predominantemente semi-diurno. De acordo com Schettini et al. (1996) a variação média de maré astronômica é de 0,8 metros, 
RIBEIRO, R.S et al. Caracterização morfológica e hidrodinâmica de praias...

sendo a máxima de $1,2 \mathrm{~m}$ em condição de sizígia. Estes autores salientam, ainda, a grande importância da maré meteorológica na dinâmica costeira regional, a qual pode ampliar os valores de nível em até um metro.

Araújo et al. (2003), com base em um ano de dados colhidos por uma boia localizada próxima à Ilha de Santa Catarina, definiram o clima de ondas na costa do estado como sendo predominado por marulhos de Sul (período de até $12 \mathrm{~s}$ e altura significativa em torno de $1,25 \mathrm{~m}$ no verão e $2 \mathrm{~m}$ no inverno) e vagas de Leste (período de $8 \mathrm{~s}$ e altura significativa de $1,25 \mathrm{~m}$ ). Os marulhos se apresentam mais frequentes nas estações de outono e inverno, enquanto que no verão foi verificado um equilíbrio entre a dominância de vagas e de marulhos.

Destaca-se, ainda, a frequente ocorrência de eventos extremos em todo o estado, sendo estes caracterizados pela conjunção de marés meteorológicas e ondas de tempestade, potencializados em condições de sizígia (RUDORFF et al., 2014).

\section{Materiais e métodos}

Apesar do senso comum, deve-se ressaltar que o volume de incidentes com banhistas nem sempre está relacionado com a energia de ondas de uma praia.

Tendo isso em consideração, a análise de perigo ao banhista foi realizada com base na metodologia proposta por Short (2002), que relaciona o estágio morfodinâmico da praia com a altura de quebra da onda. As etapas e dados envolvidos no desenvolvimento da pesquisa são apresentados na Figura 1. 
RIBEIRO, R.S et al. Caracterização morfológica e hidrodinâmica de praias...

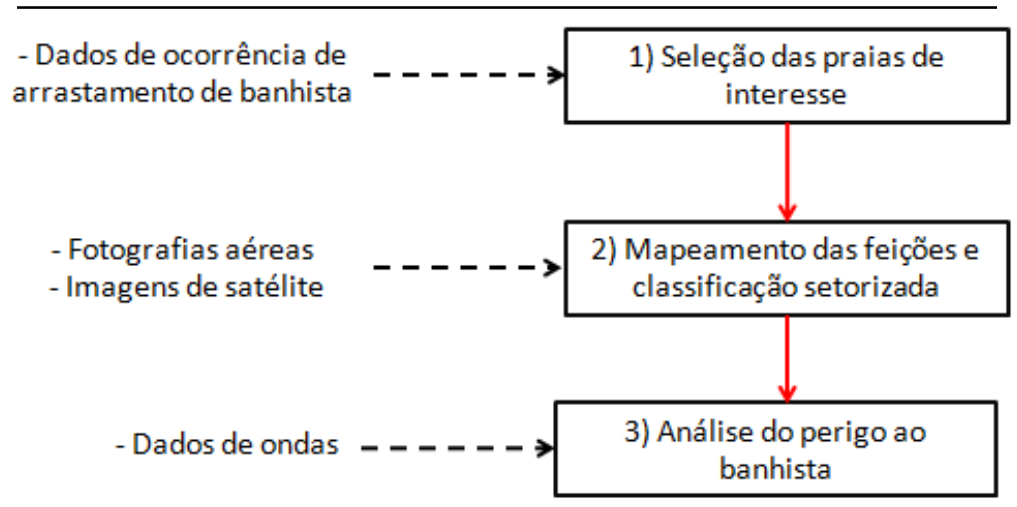

Figura 1: Etapas e dados envolvidos no desenvolvimento do trabalho.

\section{Seleção das praias}

A escolha das praias a serem estudadas foi baseada nos números de incidentes registrados nos relatórios do Corpo de Bombeiros Militar de Santa Catarina. Selecionou-se, a partir desses dados, 14 praias onde o número de ocorrências de arrastamento foi superior a 100 casos (Figura 2).

Os registros de arrastamento foram obtidos através da página do Corpo de Bombeiros. O número de ocorrências de arrastamento, afogamento com recuperação e afogamento seguido de morte (entre outros registros relevantes) para todas as cidades do estado estão disponíveis em http://www.cbm.sc.gov.br/rel_praias/. Foram utilizados os dados de arrastamento da Operação Veraneio 2012/2013.

As praias selecionadas estão localizadas nos setores central e norte da costa do Estado de Santa Catarina. Nestes setores observase a ocorrência de diversas faixas de areia limitadas por promontórios, resultando em um conjunto expressivo de praias de enseada, com extensão relativamente pequena. 
RIBEIRO, R.S et al. Caracterização morfológica e hidrodinâmica de praias...

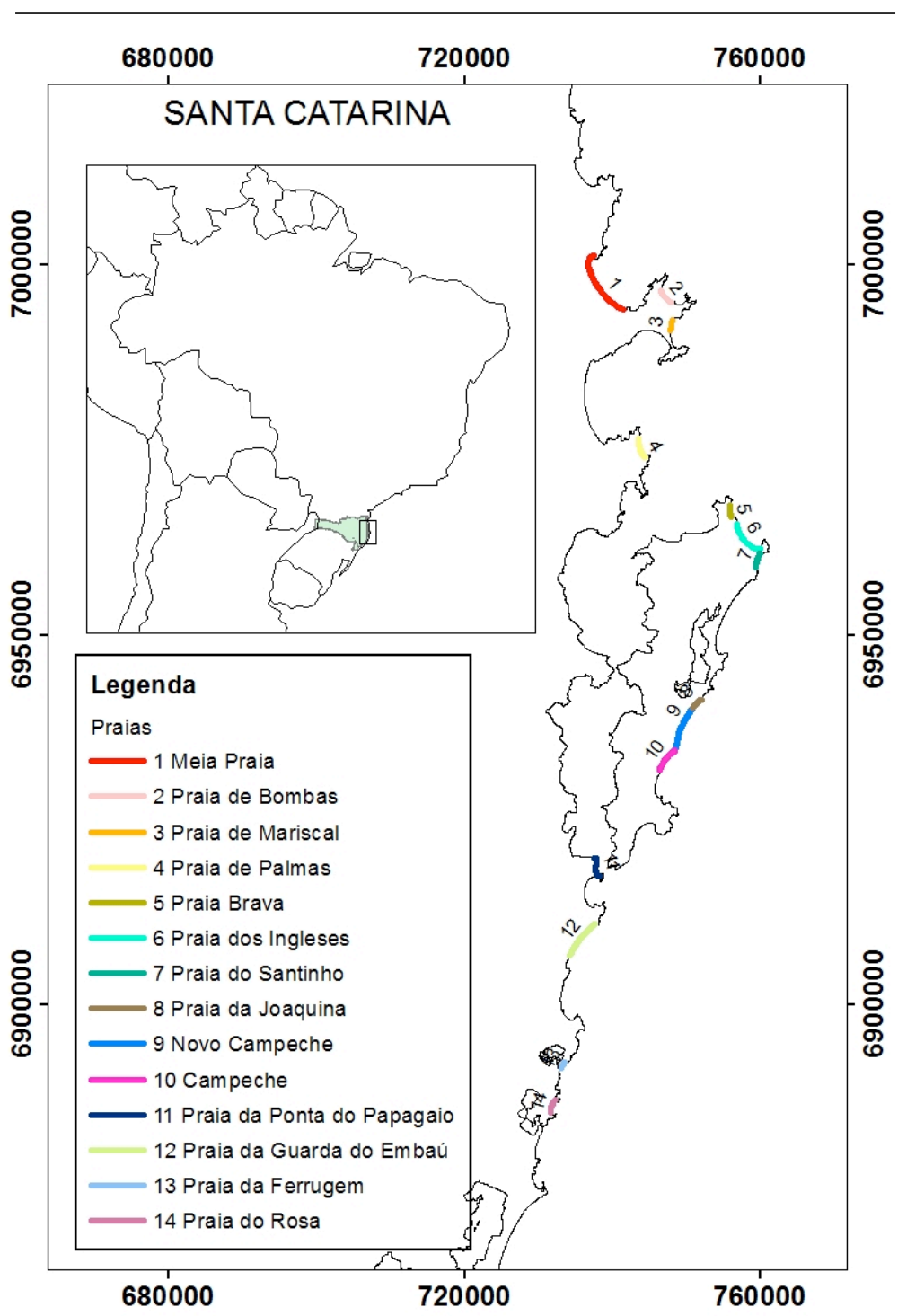

Geosul, v.30, n.60, 2015 
RIBEIRO, R.S et al. Caracterização morfológica e hidrodinâmica de praias...

\section{Figura 2: Mapa de localização das praias analisadas. Mapeamento das feições e classificação setorizada}

A classificação das praias selecionadas se deu de acordo com os estágios morfodinâmicos propostos por Wright e Short (1984). De modo a identificar as feições importantes na classificação morfodinâmica das praias (bancos, correntes de retorno, cúspides e zona de surfe) imagens de satélite das missões QuickBird e GeoEye foram utilizadas. Com base nestas, as feições foram mapeadas como apresentado no exemplo da Figura 3. Metodologia semelhante foi aplicada anteriormente por Silveira et al. (2011) para praias de São Paulo e Santa Catarina.

Algumas praias de enseada podem ter características morfodinâmicas distintas em diferentes trechos, devido à diferença de energia incidente ao longo da praia (KLEIN, 2004). O setor mais protegido da praia tende a ser refletivo por receber ondas de menor energia, ao contrário do seu trecho central, submetido a ondas com maior energia, que é mais retilíneo e se apresenta como dissipativo. Segundo Klein \& Menezes (2001) essa variação dentro da mesma praia também ocorre em função do tamanho do grão e das características geológicas e hidrológicas locais.

Levando em conta a diferenciação que ocorre nos setores de uma mesma praia optou-se por realizar a classificação das 14 praias selecionadas em 3 setores: norte, centro e sul, sempre que disponíveis dados de onda para os diferentes trechos. Desta forma, utilizando a tipologia anteriormente descrita, foram classificados os diferentes setores que compõem cada praia. Para tal, foram observadas diferenças como, por exemplo, a presença ou não de correntes, presença de zona de surfe ou altura de quebra de onda.

Também foi realizada uma análise temporal desses setores a partir da interpretação das imagens de satélites obtidas. Com isso pode-se observar a perenidade das diferentes feições observadas. A verificação da variação temporal da classificação proposta foi realizada através de imagens de satélite obtidas em três anos distintos. As imagens foram escolhidas entre o período de 2003 a 2014, dependendo das condições de cobertura de nuvens. 
RIBEIRO, R.S et al. Caracterização morfológica e hidrodinâmica de praias...

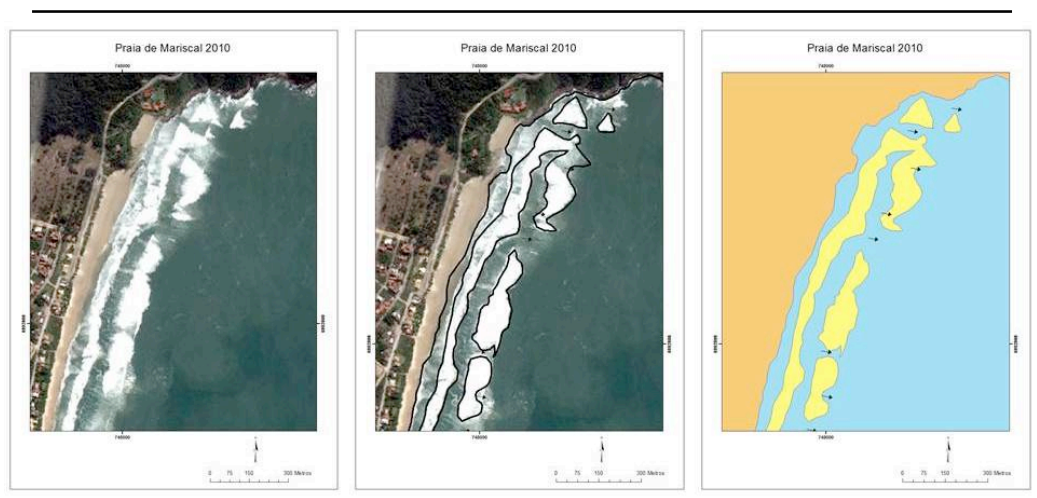

Figura 3: Exemplo de mapeamento das feições praiais (Praia do Mariscal, imagem obtida em 2010).

Para classificar as praias quanto a seu estágio morfodinâmico foram observadas, nas imagens de satélite, características morfológicas das praias que permitem distinguir os estados dissipativo, intermediário (banco e cava, bancos e correntes, bancos rítmicos e terraços de maré) e refletivo, tendo como ponto de partida o trabalho de Silveira et al. (2011):

\section{Praias dissipativas}

a) Presença de Bancos Múltiplos: a identificação dos bancos múltiplos foi feita observando-se a espuma produzida pela quebra das ondas;

b) Sistemas de bancos múltiplos gerando extensas zonas de surfe $(>100 \mathrm{~m})$

c) Zona de espraiamento extensa ( $>10 \mathrm{~m})$. Indicativo de praia com baixa declividade.

\section{Praias intermediárias}

a) Presença de Banco Longitudinal, Rítmico ou Transversal: a identificação dos bancos foi feita diretamente onde a água é pouco turva, possibilitando a visualização direta ou através de padrões de espuma resultantes da quebra das ondas. Zonas de 
RIBEIRO, R.S et al. Caracterização morfológica e hidrodinâmica de praias...

espuma com intervalos longitudinais são indícios da presença de bancos rítmicos ou transversais, enquanto padrões de quebra de ondas contínuos ao longo da costa são indicativos de bancos longitudinais.

b) Presença de cúspides e/ou mega-cúspides: as cúspides foram identificadas como sendo feições rítmicas na linha de costa de pequena escala $(<100 \mathrm{~m})$. Já as mega-cúspides foram identificadas como sendo feições rítmicas na linha de costa de grande escala $(>100 \mathrm{~m})$.

c) Presença de Correntes de Retorno: as correntes de retorno foram observadas pela lacuna na espuma gerada pela quebra e pela tonalidade da água próximo à zona de arrebentação. Com alta capacidade de transporte, a presença das correntes de retorno é associada a tonalidades similares à dos sedimentos da praia, correspondente aos sedimentos em suspensão.

\section{Praias refletivas}

a) Quebra de ondas na base da zona de espraiamento: como esse tipo de praia não apresenta bancos, a quebra de ondas só ocorre nas proximidades da zona de espraiamento, que é estreita devido à alta declividade da face praial.

b) Presença de Cúspides: as cúspides serão identificadas como sendo feições rítmicas na linha de costa com pequena escala $(<100 \mathrm{~m})$.

\section{Altura de quebra da onda}

Os dados de altura de quebra de onda foram extraídos da base de dados do programa SMC-Brasil (SMC Brasil, 2013). O SMC-Brasil faz parte do Projeto Acordo de Cooperação Técnica Internacional entre Brasil e Espanha, coordenado no Brasil pelo Ministério do Meio Ambiente e Secretaria do Patrimônio da União/ Ministério do Planejamento. Dentre os produtos do Projeto está a disponibilização de uma base de dados de ondas em pontos espaçados a cada $1 \mathrm{~km}$ em toda a costa do Brasil. 
RIBEIRO, R.S et al. Caracterização morfológica e hidrodinâmica de praias...

Os dados são resultados de reanálise de ondas e consistem em séries de 60 anos com resolução temporal horária. As reanálises foram realizadas em duas etapas, sendo a primeira global com o modelo WWIII (TOLMAN, 1991) e a segunda a propagação dos dados da primeira etapa até a costa com o modelo SWAN. A qualidade dos dados foi avaliada conforme descrito por Reguero et al. (2012).

O ideal para a análise do grau de perigo seria a utilização de dados de altura de quebra. Todavia, a base de dados do SMC Brasil não contém dados de altura de quebra, apenas valores de altura significativa de onda. Por essa razão, escolheu-se trabalhar com os pontos de ondas mais próximos a cada setor da praia.

Os dados foram acessados através da plataforma SMCTools que fornece a análise estatística de cada ponto da base. Os valores de moda de altura de onda foram obtidos a partir do histograma de distribuição de frequência em cada ponto analisado.

Com o intuito de verificar a variabilidade espacial (em uma mesma praia) do grau de perigo, mais de um ponto de ondas foi selecionado em cada praia. Sempre que possível foram escolhidos três pontos (dois nas extremidades e um na porção central). Algumas praias, todavia, apresentaram apenas dois ou um ponto de ondas sendo que, nestes casos, foram utilizados os dados disponíveis.

\section{Análise do perigo ao banhista}

A análise do perigo ao banhista foi realizada com base no quadro proposto por Short (2002) que prevê diferentes graus de perigo associados a tipos de praia distintos (Quadro 1).

O método consiste em verificar o grau de perigo de uma praia considerando seu estágio morfodinâmico e os valores de altura de quebra de onda.

O grau de perigo é calculado considerando-se que o banhista se encontra na zona de surfe e cresce com o aumento da altura da onda com a ocorrência de correntes induzidas por canais de maré, promontórios e recifes. 
RIBEIRO, R.S et al. Caracterização morfológica e hidrodinâmica de praias...

Quadro 1: Classes de perigo para os diferentes estágios morfodinâmicos e valores de altura de quebra da onda em praias oceânicas (SHORT, 2002).

PRAIAS DOMINADAS POR ONDAS

\begin{tabular}{|c|c|c|c|c|c|c|c|c|}
\hline \multirow[b]{2}{*}{ TIPO DE PRAIA } & \multicolumn{8}{|c|}{ ALTURA DE QUEBRA (m) } \\
\hline & $<0.5$ & 0.5 & 1.0 & 1.5 & 2.0 & 2.5 & 3.0 & $>3.0$ \\
\hline Dissipativa (D) & 4 & 5 & 6 & 7 & 8 & 9 & 10 & 10 \\
\hline $\begin{array}{l}\text { Bancos e Cavas } \\
\text { Longitudinais } \\
\text { (LBT) }\end{array}$ & 4 & 5 & 6 & 7 & 7 & 8 & 9 & 10 \\
\hline $\begin{array}{c}\text { Banco e Praia } \\
\text { Rítmicos (RBB) }\end{array}$ & 4 & 5 & 6 & 6 & 7 & 8 & 9 & 10 \\
\hline $\begin{array}{c}\text { Bancos e Correntes } \\
\text { Trans-versais } \\
\text { (TBR) } \\
\end{array}$ & 4 & 4 & 5 & 6 & 7 & 8 & 9 & 10 \\
\hline $\begin{array}{c}\text { Terraço de Maré } \\
\text { Baixa (LTT) }\end{array}$ & 3 & 3 & 4 & 5 & 6 & 7 & 8 & 10 \\
\hline Refletiva (R) & 2 & 3 & 4 & 5 & 6 & 7 & 8 & 10 \\
\hline $\begin{array}{c}\text { TAXA DE PERIC } \\
\text { PRAIA }\end{array}$ & O DA & & & HAVE & PA & A O & ERI & \\
\hline $\begin{array}{c}\text { Pouco perigos } \\
1-3\end{array}$ & & & & $\begin{array}{l}\text { Profu } \\
\text { fracas }\end{array}$ & & e/ol & corr & \\
\hline $\begin{array}{c}\text { Moderadamente per } \\
-6\end{array}$ & igoso: 4 & & & $\begin{array}{l}\text { Queb } \\
\text { marg }\end{array}$ & $\begin{array}{l}\text { de } \\
n(m\end{array}$ & $\begin{array}{l}\text { Ida } p \\
\text { ré alt }\end{array}$ & $\begin{array}{l}\text { xim } \\
\text { aper }\end{array}$ & \\
\hline $\begin{array}{c}\text { Altamente perig } \\
7-8\end{array}$ & oso: & & & $\begin{array}{l}\text { Corre } \\
\text { quebr }\end{array}$ & tes e & $\begin{array}{l}\text { xten } \\
\text { da }\end{array}$ & zon & \\
\hline $\begin{array}{c}\text { Extremamente per } \\
9-10\end{array}$ & igoso: & & & $\begin{array}{l}\text { Corre } \\
\text { corre }\end{array}$ & 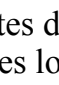 & $\begin{array}{l}\text { reto } \\
\text { gituc }\end{array}$ & & \\
\hline
\end{tabular}


RIBEIRO, R.S et al. Caracterização morfológica e hidrodinâmica de praias...

\section{Resultados e discussão}

\section{Mapeamento das feições e classificação setorizada}

As feições identificadas nas praias analisadas e a classificação adotada para cada uma delas estão apresentadas na Tabela 1.

Das praias deste trabalho a maioria, treze de catorze, apresentou estágio morfodinâmico intermediário. Apenas a Ponta do Papagaio apresentou estágio refletivo. Isso pode estar relacionado às alturas de onda que atingem o local; situada próximo à desembocadura da Baía Sul de Florianópolis, trata-se de uma praia relativamente abrigada da ação das ondas, característica típica de praias refletivas (SHORT, 1999). Nenhuma das praias selecionadas apresentou estágio morfodinâmico dissipativo.

Tabela 1: Características dos diferentes setores de praias analisados: feições da praia, classificação morfodinâmica, altura de onda e resultado da análise de perigo $(\mathrm{N}=$ setor norte, $\mathrm{C}=$ setor central, $\mathrm{S}=$ setor sul e $\mathrm{U}=$ setor único).

\begin{tabular}{|c|c|c|c|c|c|c|c|}
\hline Praia & Setor & $\begin{array}{c}\text { Zona de } \\
\text { Surfe }\end{array}$ & $\begin{array}{c}\text { Correntes } \\
\text { de Retorno }\end{array}$ & Cúspides & Class. & $\begin{array}{l}\text { Hs } \\
(\mathrm{m})\end{array}$ & $\begin{array}{c}\text { Classe } \\
\text { de } \\
\text { Perigo }\end{array}$ \\
\hline \multirow{3}{*}{ Meia Praia } & $\mathrm{N}$ & 10 a $80 \mathrm{~m}$ & 0 & $20 \mathrm{~m}$ & LTT & 0.5 & 3 \\
\hline & $\mathrm{C}$ & $10 \mathrm{a} 40 \mathrm{~m}$ & 0 & $20 \mathrm{~m}$ & LTT & 0.5 & 3 \\
\hline & $\mathrm{S}$ & 0 a $20 \mathrm{~m}$ & 0 & 10 a $25 \mathrm{~m}$ & LTT & 0.3 & 3 \\
\hline \multirow{3}{*}{ Bombas } & $\mathrm{N}$ & 30 a $60 \mathrm{~m}$ & 6 & 20 a $30 \mathrm{~m}$ & TBR & 0.75 & 5 \\
\hline & $\mathrm{C}$ & 30 a $60 \mathrm{~m}$ & 2 a 7 & 15 a $20 \mathrm{~m}$ & TBR & 0.5 & 4 \\
\hline & $\mathrm{S}$ & 20 a $50 \mathrm{~m}$ & 2 a 5 & $0115 \mathrm{~m}$ & TBR & 0.5 & 4 \\
\hline Mariscal & $\mathrm{U}$ & 30 a $200 \mathrm{~m}$ & 5 a 10 & $24 \mathrm{a} 75 \mathrm{~m}$ & LTT & 0.7 & 4 \\
\hline \multirow{3}{*}{ Palmas } & $\mathrm{N}$ & 25 a $55 \mathrm{~m}$ & 6 a 9 & $10 \mathrm{a} 40 \mathrm{~m}$ & TBR & 0.75 & 4 \\
\hline & $\mathrm{C}$ & 15 a $70 \mathrm{~m}$ & 3 a 6 & 10 a $25 \mathrm{~m}$ & TBR & 0.75 & 4 \\
\hline & $\mathrm{S}$ & 0 a $35 \mathrm{~m}$ & 0 a 3 & ausente & TBR & 0.5 & 4 \\
\hline \multirow{2}{*}{ Brava } & $\mathrm{N}$ & 20 a $90 m$ & 4 a 5 & $15 \mathrm{a} 170 \mathrm{~m}$ & TBR & 1.2 & 5 \\
\hline & $\mathrm{S}$ & $0 \mathrm{a} 90 \mathrm{~m}$ & 1 a 3 & $10 \mathrm{a} 100 \mathrm{~m}$ & TBR & 1 & 5 \\
\hline \multirow[b]{2}{*}{ Ingleses } & $\mathrm{N}$ & 30 a $75 \mathrm{~m}$ & 3 a 5 & $15 \mathrm{a} 40 \mathrm{~m}$ & LTT & 0.75 & 3 \\
\hline & $\mathrm{C}$ & 25 a $35 \mathrm{~m}$ & $1 \mathrm{a} 7$ & 10 a $20 \mathrm{~m}$ & LTT & 0.75 & 3 \\
\hline
\end{tabular}

Geosul, v.30, n.60, 2015 
RIBEIRO, R.S et al. Caracterização morfológica e hidrodinâmica de praias...

\begin{tabular}{|c|c|c|c|c|c|c|c|}
\hline & $\mathrm{S}$ & 0 a $15 \mathrm{~m}$ & 0 & 10 a $20 \mathrm{~m}$ & $\mathrm{R}$ & 0.5 & 3 \\
\hline \multirow{3}{*}{ Santinho } & $\mathrm{N}$ & 90 a $100 \mathrm{~m}$ & 3 a 4 & ausente a $120 \mathrm{~m}$ & TBR & 1 & 5 \\
\hline & $\mathrm{C}$ & 90 a $100 \mathrm{~m}$ & 2 a 4 & 50 a $100 \mathrm{~m}$ & TBR & 1.2 & 5 \\
\hline & $\mathrm{S}$ & 60 a $90 \mathrm{~m}$ & 1 a 6 & 30 a $100 \mathrm{~m}$ & TBR & 1 & 5 \\
\hline Joaquina & Ú & 50 a $180 \mathrm{~m}$ & 4 a 9 & $15 \mathrm{a} 60 \mathrm{~m}$ & TBR & 1 & 5 \\
\hline \multirow{3}{*}{$\begin{array}{l}\text { Novo } \\
\text { Campeche }\end{array}$} & $\mathrm{N}$ & 30 a $120 \mathrm{~m}$ & 1 a 5 & 50 a $200 \mathrm{~m}$ & TBR & 1 & 4 \\
\hline & $\mathrm{C}$ & 30 a $100 \mathrm{~m}$ & 1 a 5 & 30 a $160 \mathrm{~m}$ & TBR & 0.75 & 4 \\
\hline & $\mathrm{S}$ & $20 \mathrm{a} 60 \mathrm{~m}$ & 1 a 4 & 20 a $50 \mathrm{~m}$ & TBR & 0.5 & 4 \\
\hline \multirow{3}{*}{ Campeche } & $\mathrm{N}$ & $15 \mathrm{a} 100 \mathrm{~m}$ & 4 a 7 & 100 a $200 \mathrm{~m}$ & TBR & 0.75 & 4 \\
\hline & $\mathrm{C}$ & 15 a $90 \mathrm{~m}$ & 0 a 2 & 50 a $90 \mathrm{~m}$ & TBR & 1 & 4 \\
\hline & $\mathrm{S}$ & $0 \mathrm{a} 80 \mathrm{~m}$ & 2 a 7 & 20 a $90 \mathrm{~m}$ & TBR & 0.75 & 5 \\
\hline \multirow{3}{*}{$\begin{array}{l}\text { Ponta do } \\
\text { Papagaio }\end{array}$} & $\mathrm{N}$ & $0 \mathrm{a} 10 \mathrm{~m}$ & 0 a 2 & ausente a $15 \mathrm{~m}$ & LTT & 0.2 & 3 \\
\hline & $\mathrm{C}$ & 0 a $20 \mathrm{~m}$ & 0 & ausente a $60 \mathrm{~m}$ & $\mathrm{R}$ & 0.35 & 3 \\
\hline & $\mathrm{S}$ & $0 \mathrm{a} 10 \mathrm{~m}$ & 0 & 10 a $15 \mathrm{~m}$ & $\mathrm{R}$ & 0.5 & 3 \\
\hline \multirow{3}{*}{$\begin{array}{l}\text { Guarda do } \\
\text { Embaú }\end{array}$} & $\mathrm{N}$ & 40 a $100 \mathrm{~m}$ & 5 a 11 & 30 a $50 \mathrm{~m}$ & LBT & 0.8 & 5 \\
\hline & $\mathrm{C}$ & 50 a $65 \mathrm{~m}$ & 0 a 11 & $30 \mathrm{~m}$ & LBT & 0.9 & 5 \\
\hline & $\mathrm{S}$ & 30 a $50 \mathrm{~m}$ & 0 a 3 & $30 \mathrm{~m}$ & LBT & 0.75 & 5 \\
\hline Ferrugem & $\mathrm{U}$ & 90 a $150 \mathrm{~m}$ & 5 a 9 & $30 \mathrm{a} 40 \mathrm{~m}$ & LBT & 1.25 & 5 \\
\hline Rosa & $\mathrm{U}$ & 100 a $200 \mathrm{~m}$ & 10 a 13 & 25 a $50 \mathrm{~m}$ & RBB & 0.75 & 5 \\
\hline
\end{tabular}

A maioria dos trabalhos anteriores realizou a classificação de diferentes praias do Estado de Santa Catarina em apenas 3 estágios, considerando todas as praias intermediárias como uma única classificação.

Klein et al. (2005) também classificaram algumas das praias aqui analisadas. Naquele trabalho, Bombas, Brava, Ingleses e Campeche foram consideradas praias intermediárias, estando de acordo com a classificação apresentada neste estudo. No entanto, os autores consideraram Meia Praia e Santinho como dissipativas. Ressalta-se, entretanto, que estes não as associaram a praias dissipativas expostas. Klein e Menezes (2001) concluíram que as praias protegidas de Santa Catarina têm sua forma e declividade influenciadas pela fonte do sedimento e pelo tamanho do grão disponível. Tendo-se isso em consideração, há divergência sobre a plena existência de praias do tipo terraço de maré baixa, que teoricamente deveriam apresentar ambos os 
RIBEIRO, R.S et al. Caracterização morfológica e hidrodinâmica de praias...

perfis: convexo na preamar e côncavo na baixa mar. No presente trabalho, para fins práticos, foi adotada a terminologia "terraço de maré baixa" para essas praias com areia fina a muito fina e perfil de baixa declividade e praial côncavo. Todavia admite-se que deva ocorrer sua reclassificação a partir do desenvolvimento de estudos mais aprofundados sobre essa questão.

Silveira et al. (2011), utilizando imagens de satélite também classificaram as praias Bombas, Mariscal, Palmas, Santinho, Joaquina, Campeche, Guarda do Embaú e Ferrugem como praias intermediárias. No entanto, houve diferenças entre a classificação dos autores com relação à classificação apresentada neste trabalho. As praias de Meia Praia e Joaquina foram classificadas como dissipativas (ver discussão do parágrafo anterior), enquanto o mapeamento do presente estudo indicou que estas praias apresentam estágio intermediário (terraço de maré baixa e bancos e correntes transversais, respectivamente). $\mathrm{Na}$ praia de Ingleses a análise das feições no setor sul da praia não indicou em nenhuma das imagens a presença de banco ou corrente de retorno. Isso sugere que este trecho se encontra em estágio refletivo e não terraço de maré baixa como sugerido na classificação preliminar. A classificação proposta concorda com Faraco (2003) que classificou a praia como Intermediária e com Silveira et al. (2011) que classificou a praia como refletiva. A associação da praia dos Ingleses ao estágio morfodinâmico intermediário e também ao refletivo pode ser explicada pelo fato de terraço de maré baixa apresentar características refletivas na maré alta e dissipativas na maré baixa (FARACO, 2003).

Outra ressalva é apontada por Oliveira (2009), que em seu trabalho encontrou em uma praia intermediária do tipo terraço de maré baixa uma zona de sombra apresentando baixa declividade e areia fina a muito fina, características essas de praias dissipativas, concluindo assim que em zonas de sombra praias de areia fina podem apresentar estágios morfodinâmicos próximos ao estágio refletivo.

A classificação setorizada também apresentou setores diferentes ocorrendo na praia da Ponta do Papagaio, onde observou-se a presença de uma pequena zona de surfe na porção norte da praia. Este 
RIBEIRO, R.S et al. Caracterização morfológica e hidrodinâmica de praias...

setor apresenta estágio intermediário do tipo terraço de maré baixa. Já na porção sul, a praia foi classificada como refletiva.

A praia da Guarda do Embaú, apesar de ser considerada inteiramente do tipo bancos e cavas longitudinais, na imagem de satélite de 2006 apresentou a presença de bancos com características rítmicas no setor norte. Isso pode estar relacionado ao clima de ondas do período em que a imagem foi adquirida.

Silveira et al. (2011) alertam que a resolução das imagens e as condições atmosféricas do momento da captura da imagem influenciam a interpretação e consequentemente a classificação dos estágios morfodinâmicos de praia. Algumas características físicas da praia podem ficar não aparentes como os bancos de areia e também eventuais condições extremas como ressacas podem condicionar a erros de classificação, gerando assim a necessidade de análises temporais a fim de evitar esses tipos de problemas de interpretação das imagens. Isto significa que as características morfodinâmicas podem ser influenciadas por condições meteorológicas e/ou oceanográficas específicas (variações na altura de onda, maior turbidez na água, etc.), alterando assim, o resultado da interpretação das imagens.

\section{Análise do perigo ao banhista}

\section{Altura de quebra da onda}

Os valores de moda da altura de onda obtidos a partir da base de dados do SMC Brasil para cada setor das praias estão apresentados na Tabela 1 . Os valores de Hs obtidos variaram de 0,2 a 1,25. Os menores valores de altura significativa ocorreram na praia da Ponta do Papagaio e os maiores valores nas praias Brava, Ingleses, Joaquina e Ferrugem. Houve grande diferença entre as profundidades em que os dados foram obtidos. Como dito anteriormente, foram escolhidos os pontos mais próximos a cada praia. No entanto, não foi possível padronizar a profundidade em que estes dados foram obtidos, uma vez que se dependia dos limites da base de dados do SMC Brasil. Ainda assim, os valores de altura de onda extraídos dos SMC conferem com os valores de altura de quebra de outros trabalhos, como na praia de Ingleses por exemplo. Faraco (2003) fez a análise visual das alturas de 
RIBEIRO, R.S et al. Caracterização morfológica e hidrodinâmica de praias...

quebra da praia de Ingleses ao longo de vários meses e os valores obtidos foram semelhantes aos apresentados aqui: até $0,5 \mathrm{~m}$ no setor sul e de 0,5 a 1,20 $\mathrm{m}$ na porção norte.

\section{Grau de perigo ao banhista}

Os valores de grau de perigo em cada setor das praias analisadas são apresentados na Tabela 1 .

De modo geral, as praias apresentaram graus de perigo de baixo a moderado, considerando toda a escala prevista pelo método adotado. Isso se deve ao fato de que as praias foram selecionadas de acordo com a ocorrência de arrastamento e não com base nos extremos de altura de onda e estágio morfodinâmico, que são as variáveis consideradas na metodologia. Hoefel (1998) discute que as maiores ocorrências podem estar relacionadas a praias com maior número de banhistas, que, via de regra, são as que oferecem segurança. Desta forma, praias notadamente perigosas, submetidas a ondas de grande altura, tendem a ser evitadas.

Os graus de perigo variaram de 3 a 5 entre as praias deste estudo. Maior grau de perigo foi observado em Bombas (setor norte), Brava, Santinho, Joaquina, setor sul da praia do Campeche, norte da Praia do Novo Campeche, Guarda do Embaú, Ferrugem e Rosa, todas com alturas de onda maiores que $0,75 \mathrm{~m}$. Ao mesmo tempo, quase todas (com exceção da praia do Rosa) apresentam estágio intermediário do tipo bancos transversais e correntes de retorno que, de acordo com Short (2002), é o tipo de praia que apresenta maiores ocorrências de afogamento devido aos perigos existentes.

Os menores graus de perigo foram verificados em Meia Praia, Ingleses e Ponta do Papagaio. Meia Praia e Ponta do Papagaio foram as praias que apresentaram menores valores de altura de onda. Meia Praia e parte dos Ingleses foram classificadas como Terraço de Maré Baixa, assim o setor Norte da Ponta do Papagaio. De acordo com Short (2002), estes são os tipos praia mais seguros desde que apresentem alturas menores que $1 \mathrm{~m}$, caso de Ingleses e Meia Praia.

As praias de Bombas e Brava foram aquelas que apresentaram maior número de ocorrências de arrastamento e estão entre as praias 
RIBEIRO, R.S et al. Caracterização morfológica e hidrodinâmica de praias...

com maior grau de perigo. No entanto, Novo Campeche também apresentou maior grau de perigo e foi a praia com menos ocorrências entre as praias selecionadas. Isso demonstra que a metodologia adotada quantifica o perigo e não o risco, uma vez que não considera o fator homem na análise. Isso reforça o que foi apresentado por Short (2002) ao propor a metodologia, dizendo que a mesma só é válida se considerar os banhistas na praia. Da mesma forma, de acordo com Mocellin (2006), uma praia altamente perigosa pode não apresentar risco algum se não for frequentada por banhistas.

Desta forma, estudos sobre a quantidade e caracterização das pessoas que frequentam determinada praia seriam necessários para análises dos riscos aos banhistas.

\section{Conclusões}

Neste trabalho foram apresentados graus de perigo ao banhista em catorze praias da costa de Santa Catarina, Região Sul do Brasil. Dentre as praias analisadas houve predomínio do estágio intermediário, com apenas uma praia refletiva e nenhuma praia dissipativa. As praias do Estado de Santa Catarina apresentaram graus baixos a moderados de perigo.

Os maiores valores de perigo estiveram, de modo geral, relacionados a praias do tipo bancos transversais e correntes de retorno. Já os menores valores foram associados a praias do tipo terraço de maré baixa, concordando com a literatura consultada.

É importante ressaltar que a metodologia adotada para as classificações morfológicas apresenta limitações, uma vez que o mapeamento das feições é baseado em imagens de satélite que representam o estado da praia conforme as ondas no dia e no período imediatamente anterior à aquisição da imagem. A fim de minimizar esse problema, os mapeamentos foram realizados a partir de imagens adquiridas em três datas diferentes. Ainda assim não é possível afirmar que as praias não apresentam estágios morfodinâmicos diferentes em outras condições. Da forma semelhante, o estágio morfodinâmico pode variar ao longo do arco praial, tendo isso sido considerado na 
RIBEIRO, R.S et al. Caracterização morfológica e hidrodinâmica de praias...

presente análise, que buscou avaliar as características morfológicas e de incidência de ondas em três trechos de cada praia.

A comparação com trabalhos anteriores mostrou que a metodologia utilizada representou bem os estágios morfodinâmicos e os graus de perigo aos quais as praias selecionadas neste estudo estão submetidas. Estudos complementares, que incorporem dados de frequência dessas praias, permitirão um diagnóstico mais preciso do perigo ao qual os banhistas se encontram submetidos nestes locais.

\section{Agradecimentos}

Os autores expressam seus agradecimentos ao Professor Andrew Short pelas sugestões dadas ao longo do desenvolvimento da pesquisa e à CAPES pela concessão de bolsa de mestrado ao primeiro autor deste trabalho.

\section{Referências bibliográficas}

ARAÚJO, C. E. S., FRANCO, D., MELO FILHO, E., PIMENTA, F. Wave regime characteristics of southern Brazilian coast. $6^{\circ}$ International Conference on Coastal and Port Engineering in Developing Countries, COPEDEC. Colombo, Sri Lanka, Paper no97, 15p. 2003.

FARACO, K.R. Comportamento morfodinâmico e sedimentológico da Praia dos Ingleses - Ilha de Santa Catarina - SC, durante o período de 1996 - 2001. Dissertação (Mestrado em Geografia) - Universidade Federal de Santa Catarina. Florianópolis, 120p. 2003.

HOEFEL, F. G. Morfodinâmica de praias arenosas oceânicas: uma revisão bibliográfica. Itajaí: Editora da Univali, 1998.

HOEFEL, F.G. \& A.H.F. Klein - Environmental and Social Decision Factors of Beach Safety in the Central Northern Coast of Santa Catarina, Brazil. Notas Técnicas Facimar. v2: pp155-166, 1998. 
RIBEIRO, R.S et al. Caracterização morfológica e hidrodinâmica de praias...

KLEIN, A.H.F. Morphodynamics of Headland Bay Beaches Examples from the coast of Santa Catarina State, Brazil. $257 \mathrm{f}$. Tese - Universidade de Algarve. Faro: 2004.

KLEIN, A.H.F., MEDEIROS, R. P. Relatório Final Projeto segurança nas praias Verão 1997/1998.

KLEIN, A.H.F., MENEZES, J. T. Beach morphodynamics and profiles sequence for a headland bay coast. Journal of Coastal Reserch. Vol 17 (4), p 198-215, 2001.

KLEIN, A. H. F.; MOCELliM O.; MENEZES, J. T.; BERRIBILLI, M.; VINTÉM, G.; DAFFERNER, G.; DIEHL, F. L.; SPERB, R. M.; SANTANA, G. G. Beach Safety Management on the Coast of Santa Catarina, Brazil. Z. Geomorph. v.141, p.47-58, 2005.

MOCELlin, O. Determinação do Nível de Risco Público ao Banho de Mar das Praias Arenosas do Litoral Centro Norte de Santa Catarina. Dissertação (Mestrado em Ciência e Tecnologia Ambiental) - Universidade do Vale do Itajaí, Itajaí, 2006.

OLIVEIRA U. R., BARLETTA R. C., PEIXOTO J. R. V., HORN FILHO N. O. Características morfodinâmicas da praia do Pântano do Sul, Ilha de Santa Catarina, Brasil. Pesquisas em Geociências, 36 (3): 237-250, mai./ago. 2009.

REGUERO, B. G.; MENÉNDEZ, M.; MÉNDEZ, R.; MÍNGUEZ, R. \& LOSADA, I. J. A Global Ocean Wave (GOW) calibrated reanalysis from 1948 onwards. Coastal Engineering. v. 65, pp. 38-55. 2012.

RUDORFF, F. M., BONETTI FILHO, J., MORENO, D. A., OLIVEIRA, C. A. F, MURARA, P. G. Maré de Tempestade. In: Herrmann, M. L. P. Atlas de Desastres Naturais do Estado de Santa Catarina: período de 1980 a 2010. 2. Ed. IHGSC/Cadernos Geográficos, Florianópolis, p. 151-154. 2014. 
RIBEIRO, R.S et al. Caracterização morfológica e hidrodinâmica de praias...

SCHETTINI, C. A. F.; CARVALHO, J. L. B.; JABOR, P. Comparative hydrology and suspended matter distribution of four estuaries in Santa Catarina State - Southern Brazil. In: Anais, Workshop on Comparative Studies of Temperate Coast Estuaries. Bahia Blanca, 1996.

SHORT, A.D. Handbook of Beach and Shoreface Morphodynamics. New York: John Wiley e Sons, 1999.

SHORT, A. D. Beaches of the New South Wales Coast: a guide to their nature, characteristics, surf and safety. Sydney, Australia, 358p. 2000.

SHORT, A., D. Australian Beach Safety \& Management Program Surf Life Saving Australia, 2002. Disponível em: http:// surfrescue.com.au . Acesso em 28 jul 2014.

SILVEIRA, L. F.; KLEIN, A. H. F.2 \& TESSLER, M. G. Classificação Morfodinâmica das Praias do Estado de Santa Catarina e do Litoral Norte do Estado de São Paulo Utilizando Sensoriamento Remoto. Brazilian Journal of Aquatic Science and Technology, v. 15, n.2, p.13-28. 2011.

SMC BRASIL - Diálogos Brasil-Espanha: Sistema de Modelagem Costeira, Resumo Executivo. In: Seminário Internacional Brasil-Espanha: A Experiência Espanhola e a Aplicação do SMC Brasil no Apoio à Gestão da Costa Brasileira, 2. Brasília: IABS. 2013.

SPILMAN, D. Afogamento. Revista Brasileira de Medicina Esportiva. v. 6, n. 4, p. 131-144. 2000.

TOLMAN, H.L. A third-generation model for wind waves on slowly varying unsteady and inhomogeneous depths and currents. Journal of Physical Oceanography.v.21, pp. 782-797, 1991. 
RIBEIRO, R.S et al. Caracterização morfológica e hidrodinâmica de praias...

\author{
WRIGHT, L.D. and SHORT, A.D. Morphodynamic variability of \\ surf zones and beaches: a synthesis. Marine Geology, v. 56, p \\ 93-118. 1984
}

Recebido em agosto de 2014 Aceito em setembro de 2015 